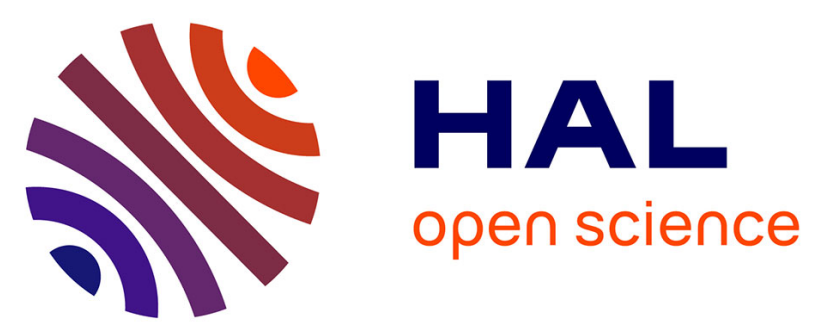

\title{
GAS FLOWS WITH HEAT TRANSFER IN MICRO CHANNELS: CLARIFICATIONS ABOUT THE NUSSELT NUMBER
}

Xavier Nicolas, Eric Chénier, Chahinez Tchekiken, Guy Lauriat

\section{- To cite this version:}

Xavier Nicolas, Eric Chénier, Chahinez Tchekiken, Guy Lauriat. GAS FLOWS WITH HEAT TRANSFER IN MICRO CHANNELS: CLARIFICATIONS ABOUT THE NUSSELT NUMBER. CHT17 ICHMT International Symposium on Advances in Computational Heat Transfer, May 2017, Napoli, Italy. hal-01544307

\author{
HAL Id: hal-01544307 \\ https://hal.science/hal-01544307
}

Submitted on 21 Jun 2017

HAL is a multi-disciplinary open access archive for the deposit and dissemination of scientific research documents, whether they are published or not. The documents may come from teaching and research institutions in France or abroad, or from public or private research centers.
L'archive ouverte pluridisciplinaire HAL, est destinée au dépôt et à la diffusion de documents scientifiques de niveau recherche, publiés ou non, émanant des établissements d'enseignement et de recherche français ou étrangers, des laboratoires publics ou privés. 


\title{
GAS FLOWS WITH HEAT TRANSFER IN MICRO CHANNELS: CLARIFICATIONS ABOUT THE NUSSELT NUMBER
}

\author{
Xavier Nicolas ${ }^{\S}$, Eric Chénier, Chahinez Tchekiken, Guy Lauriat \\ Université Paris Est Marne-la-Vallée, MSME UMR 8208 CNRS, \\ 5 boulevard Descartes, 77454 Marne-La-Vallée Cedex 2, France \\ ${ }^{\S}$ Correspondence author. Email: Xavier.Nicolas@u-pem.fr
}

\begin{abstract}
This paper deals with the modelling of weakly rarefied and dilute gas flows in heated micro channels by the continuum approach, valid for Knudsen numbers smaller than about 0.1. The first order slip and thermal jump model usually used for the forced convection of gas flows in long micro channels between two infinite plates is discussed. Indeed, in the huge literature related to this subject, it appears that simplified models are often used without justifying them and recurrent errors propagate from one paper to the other. The erroneous models particularly concern the heat transfer analysis and the energy equation. The compatibility of the pressure work and viscous dissipation in the energy equation with the power of the viscous forces at the walls and the choice of an appropriate Nusselt number are particularly discussed. Our aim is to provide a consistent model for gaseous microflows and the linked heat transfer. Then, a dimensional and asymptotic analysis is performed in the context of long micro channels. An analytical solution for the temperature field and the Nusselt number is proposed in the case of a compressible gas flow in a long micro-channel maintained at a constant wall temperature. This solution is compared with the numerical solution of the full model taking into account the first order slip and thermal jump conditions at the walls, the power of the viscous forces in the wall heat flux, the thermal creep term, the pressure work and the viscous dissipation in the bulk. The vanishing values of the Nusselt number measured in the experiments by Demsis et al. (2009, 2010) are explained for the first time.
\end{abstract}

\section{INTRODUCTION}

Due to the increasing development of MEMS (Micro Electro Mechanical Systems), the study of liquid or gas flows and heat transfer in channels or ducts whose hydraulic diameter, $D_{h}$, is of the order of a few microns (say 1 to $100 \mu \mathrm{m}$ ), has given rise to a considerable amount of works over the past twenty years. For gas flows at the microscopic scale, some observed phenomena require specific models [Gadel Hak 1999, Karniadakis et al. 2005]. In a layer called the Knudsen layer, whose thickness is of the order of $\mathrm{O}(\lambda)$ from the wall, where $\lambda$ is the mean free path of the gas molecules, a state of local thermodynamic non-equilibrium exists. It results in non-linear mean velocity profiles and relations between stress and strain rates. From the continuum mechanics point of view, at the micro channel scale, when the Knudsen number is such that $0.001<K n=\lambda / D_{h}<0.1$, these phenomena translate into a non-vanishing slip velocity, a temperature jump at the wall and a phenomenon called "thermal creep" [Sharipov 2011]. The consequences of these phenomena on the macroscopic quantities such as the mass flow rate, the friction factor, the bulk temperature and the wall heat flux can be significant 
[Lockerby et al. 2005] and must be taken into account in the modelling of the convective heat transfer in MEMS with gas flows because they may have antagonistic effects on heat transfer.

For gas flows in micro channels of large aspect ratio, $L / D_{h}$, and typical hydraulic diameter $1 \mu \mathrm{m} \leq D_{h} \leq$ $10 \mu \mathrm{m}$ and length $100 \mu \mathrm{m} \leq L \leq 1 \mathrm{~mm}$, submitted to a moderate heating of the walls and to pressure variations between the inlet and the outlet of the channel of the order of one bar to a few bars, the conversion of the mechanical work of the viscous forces into internal energy and heat is very important. It can also be shown that the Mach number, $M a=u /(\gamma r T)^{1 / 2}$, and the Brinkman number, $\mathrm{Br}$ $=\mu u^{2} / k \Delta T$, can reach or exceed 1 and the Reynolds number, $R e=u D_{h} / v$, and Péclet number, $P e=$ $u D_{h} / \alpha$, can vary between $10^{-2}$ and $10^{2}$. Accordingly, modelling this type of flow requires to take into account a wide range of physical phenomena: dynamic slip, temperature jump, thermal creep, gas compressibility, pressure work, viscous dissipation, work of the viscous forces at the wall, variation of the physical properties with temperature, conjugate heat transfer, relative roughness of the walls ... A bibliographic review of the numerous studies on this subject has shown that all these effects are never taken into account simultaneously in the numerical models used.

One objective of this paper is thus to study the influence of different phenomena described above in the case of gas flows in 2D micro channels, heated or not. The analysis will particularly focus on the thermal aspects: we will analyse the pressure work (PW) and viscous dissipation (VD) in the energy equation and the power of the viscous stress (VSP) in the wall thermal boundary conditions on the evolution of the temperature field and the Nusselt number. A dimensional and asymptotic analysis will be performed to highlight the influence of each of these terms and potentially to simplify the mathematical model. Then the analytical solution of the simplified model established by Caï et al. [2007] for compressible gas convection in isothermal wall micro-channels is reminded. It is used to explain the vanishing values of the Nusselt number measured in the experiments by Demsis et al. $(2009,2010)[8,9]$ and not explained till now. The analytical solutions of this simplified formulations is then compared with the numerical solutions of the full model.

\section{GEOMETRY AND MATHEMATICAL MODEL}

Geometry We consider gas flows between two parallel smooth walls of length $L$ in the $x$-direction, separated by a distance $H$ in the transverse $y$-direction. The hydraulic diameter is $D_{h}=2 H$. The flow is generated by a pressure difference, $p_{\text {in }}-p_{\text {out }}>0$, between the inlet and outlet sections. The fluid is considered as an ideal gas with variable physical properties. The flow is assumed to be twodimensional, steady, compressible, laminar and symmetrical through the horizontal mid-plane (see Figure 1).

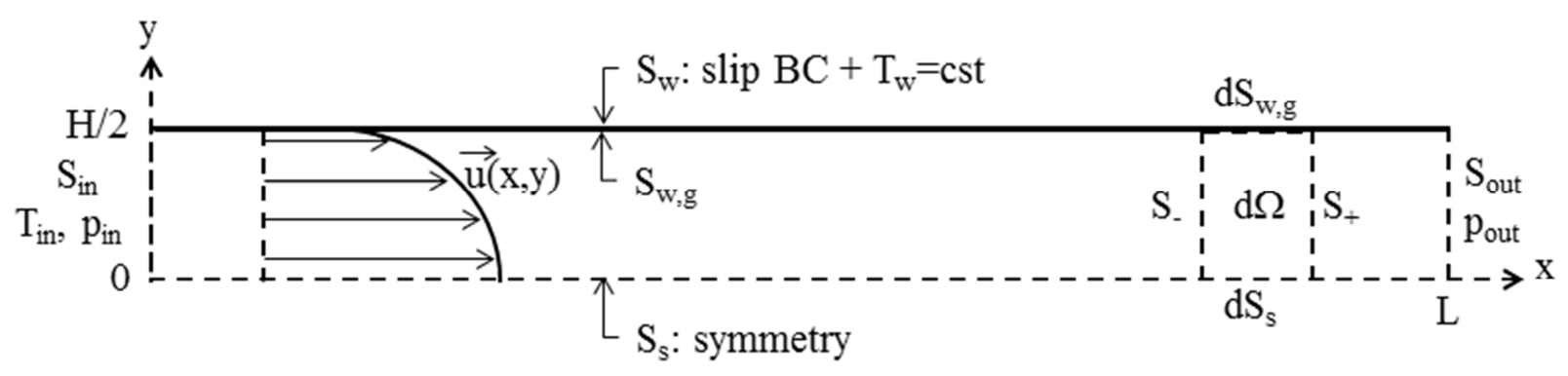

Figure 1. Geometry and boundary conditions for gas flows in 2D heated micro channels

Governing equations The governing equations are the mass, momentum and energy conservation equations in which the volume forces are neglected with respect to the viscous forces because the 
surface effects prevail in microfluidic. By denoting $(u, v)$ the velocity components in directions $(x, y)$ and $p$ and $T$ the local the pressure and temperature, the equations in Cartesian coordinates write:

$$
\begin{gathered}
\frac{\partial \rho u}{\partial x}+\frac{\partial \rho v}{\partial y}=0 \\
\frac{\partial \rho u u}{\partial x}+\frac{\partial \rho u v}{\partial y}=-\frac{\partial p}{\partial x}+\frac{\partial}{\partial x}\left[2 \mu\left(\frac{\partial u}{\partial x}-\frac{1}{3}\left(\frac{\partial u}{\partial x}+\frac{\partial v}{\partial y}\right)\right)\right]+\frac{\partial}{\partial y}\left[\mu\left(\frac{\partial u}{\partial y}+\frac{\partial v}{\partial x}\right)\right] \text { (2) } \\
\frac{\partial \rho u v}{\partial x}+\frac{\partial \rho v v}{\partial y}=-\frac{\partial p}{\partial y}+\frac{\partial}{\partial x}\left[\mu\left(\frac{\partial u}{\partial y}+\frac{\partial v}{\partial x}\right)\right]+\frac{\partial}{\partial y}\left[2 \mu\left(\frac{\partial v}{\partial y}-\frac{1}{3}\left(\frac{\partial u}{\partial x}+\frac{\partial v}{\partial y}\right)\right)\right] \text { (3) } \\
C_{p}\left(\frac{\partial \rho u T}{\partial x}+\frac{\partial \rho v T}{\partial y}\right)=\left[\frac{\partial}{\partial x}\left(k \frac{\partial T}{\partial x}\right)+\frac{\partial}{\partial y}\left(k \frac{\partial T}{\partial y}\right)\right]+\left(u \frac{\partial p}{\partial x}+v \frac{\partial p}{\partial y}\right)+\overline{\bar{\tau}}: \nabla \vec{v} \text { (4) }
\end{gathered}
$$

where $\rho$ is the density, $\mu$ the dynamic viscosity, $C_{p}$ the specific heat, $k$ the thermal conductivity and the viscous dissipation is equal to:

$$
\overline{\bar{\tau}}: \nabla \vec{v}=\mu\left\{2\left[\left(\frac{\partial u}{\partial x}\right)^{2}+\left(\frac{\partial v}{\partial y}\right)^{2}\right]+\left(\frac{\partial u}{\partial y}+\frac{\partial v}{\partial x}\right)^{2}-\frac{2}{3}\left(\frac{\partial u}{\partial x}+\frac{\partial v}{\partial y}\right)^{2}\right\}
$$

The ideal gas law closes this system, with $r$ the specific ideal gas constant:

$$
\rho(p, T)=\frac{p}{r T}
$$

Boundary conditions The associated boundary conditions are the following. At the inlet section $x=0$, uniform pressure and temperature are applied:

$$
p(0, y)=p_{i n}, \quad \frac{\partial u}{\partial x}(0, y)=0, \quad v(0, y)=0, \quad T(0, y)=T_{\text {in }}
$$

At the outlet section $x=L$, the pressure is fixed and fully developed flow conditions are applied:

$$
p(L, y)=p_{\text {out }}, \quad \frac{\partial u}{\partial x}(L, y)=0, \quad \frac{\partial v}{\partial x}(L, y)=0, \quad \frac{\partial T}{\partial x}(L, y)=0
$$

Symmetry boundary conditions are applied on the channel axis at $y=0$ :

$$
\frac{\partial u}{\partial y}(x, 0)=0, \quad v(x, 0)=0, \quad \frac{\partial T}{\partial y}(x, 0)=0
$$

On the gas side of the impermeable wall at $y=H / 2$ (denoted by the subscript $g$ below), zero normal velocity is applied and, for the tangential velocity, the rarefaction effects are modelled using the firstorder Maxwell slip velocity and thermal creep boundary conditions (see Zhang et al. [2012] and Kandlikar [2013]):

$$
\begin{gathered}
u_{g}(x)=u(x, H / 2)=\underbrace{-\left.\sigma_{\mu} \lambda_{g} \frac{\partial u}{\partial y}\right|_{g}}_{1^{\text {st }} \text { order slip velocity }}+\underbrace{\left.\sigma_{\theta} \frac{\mu_{g}}{\rho_{g} T_{g}} \frac{\partial T}{\partial x}\right|_{g}}_{\text {thermal creep }} \\
v_{g}(x)=v(x, H / 2)=0
\end{gathered}
$$

where $\lambda_{g}$ is the mean free path at the wall defined as: 


$$
\lambda_{g}=\frac{\mu_{g}}{p_{g}} \sqrt{\frac{\pi r T_{g}}{2}}=\mu_{g} \sqrt{\frac{\pi}{2 \rho_{g} p_{g}}}
$$

and $\sigma_{\mu}$ and $\sigma_{\theta}$ are the viscous and thermal slip coefficients [Sharipov 2011]. In the present numerical applications, $\sigma_{\mu}=\sigma_{\theta}=1$ which corresponds to fully accommodating walls (diffuse scattering walls).

For a channel with a uniform wall temperature $T_{w}$, the thermal boundary condition writes:

$$
T_{g}(x)=T(x, H / 2)=T_{w}-\underbrace{\left.\xi_{T} \lambda_{g} \frac{\partial T}{\partial y}\right|_{g}}_{1^{\text {st }} \text { order temperature jump }}
$$

where $\xi_{T}$ is the temperature jump coefficient [Sharipov 2011]. In the present applications, $\xi_{T}=2$.

When the Knudsen number and the wall slip are not zero, the total heat flux $q_{w}$ at the walls must be calculated as:

$$
q_{w}=\underbrace{\left.k_{g} \frac{\partial T}{\partial y}\right|_{g}}_{\text {diffusive flux }}+\underbrace{\left.\mu_{g} u_{g} \frac{\partial u}{\partial y}\right|_{g}}_{\text {viscous stress power }}
$$

where $k_{g}(x)=k\left(T_{g}(x)\right)$ and $\mu_{g}(x)=\mu\left(T_{g}(x)\right)$ are the gas conductivity and viscosity at $T_{g}(x)$. The expression (14) of the wall heat flux was presumably introduced by Maslen [1958] then used by Sparrow and Lin [1962]. Since then, only a few authors have taken into account the power of the viscous stress at the wall (second R.H.S. term) in their analysis of heat transfer. So, as most of the authors neglected this contribution, it was eventually forgotten. Hong and Asako [2010] reiterated its importance in a recent paper. We are going to analyse this contribution and show that it allows to recover the experimental results on very low values of the Nusselt numbers by Demsis et al. [2009, 2010].

\section{DIMENSIONAL ANALYSIS}

Reference quantities The equations are made dimensionless using the following variables:

$$
\begin{gathered}
X=x / L, Y=y / D_{h}, \quad U=u / \bar{u}_{i n}, V=v / \bar{u}_{i n}, P=\frac{p-p_{\text {in }}}{\Delta P_{\text {ref }}}, \theta=\frac{T-T_{\text {in }}}{\Delta T_{\text {ref }}} \\
\rho^{*}=\rho / \rho_{\text {in }}, \mu^{*}=\mu / \mu_{i n}, C_{p}^{*}=C_{p} / C_{p, i n}, \quad k^{*}=k / k_{i n}
\end{gathered}
$$

where $\bar{u}_{i n}, \rho_{i n}, p_{i n}$ and $T_{i n}$ are the mean velocity, density, pressure and temperature in the inlet section. $\mu_{i n}, C_{p, i n}$ and $k_{i n}$ are the inlet viscosity, specific heat and thermal conductivity evaluated at $T_{i n}$. In micro channels, the streamwise pressure gradient balances the viscous term, so the equality of these two terms in the Navier-Stokes equation allows defining the reference pressure difference as:

$$
\Delta P_{r e f}=\frac{\mu_{i n} \bar{u}_{i n}}{\epsilon D_{h}}
$$

where $\varepsilon=D_{h} / L$ is a small parameter for most micro channels. For a uniform wall temperature, $T_{w}$, the reference temperature difference is chosen as:

$$
\Delta T_{r e f}=T_{w}-T_{i n}
$$


Dimensionless equations and boundary conditions After introducing the dimensionless variables, the governing equations (1)-(6) take the following form:

$$
\begin{gathered}
\epsilon \frac{\partial \rho^{*} U}{\partial X}+\frac{\partial \rho^{*} V}{\partial Y}=0 \\
\epsilon \frac{\partial \rho^{*} U U}{\partial X}+\frac{\partial \rho^{*} U V}{\partial Y}=-\frac{1}{R e_{i n}} \frac{\partial P}{\partial X}+\frac{1}{R e_{i n}}\left\{\frac{\partial}{\partial X}\left[2 \mu^{*}\left(\epsilon^{2} \frac{\partial U}{\partial X}-\frac{1}{3}\left(\epsilon^{2} \frac{\partial U}{\partial X}+\epsilon \frac{\partial V}{\partial Y}\right)\right)\right]+\right. \\
\left.\frac{\partial}{\partial Y}\left[\mu^{*}\left(\frac{\partial U}{\partial Y}+\epsilon \frac{\partial V}{\partial X}\right)\right]\right\} \\
\epsilon \frac{\partial \rho^{*} U V}{\partial X}+\frac{\partial \rho^{*} V V}{\partial Y}=-\frac{1}{\epsilon R e_{i n}} \frac{\partial P}{\partial Y}+\frac{1}{R e_{i n}}\left\{\frac{\partial}{\partial X}\left[\mu^{*}\left(\epsilon \frac{\partial U}{\partial Y}+\epsilon^{2} \frac{\partial V}{\partial X}\right)\right]+\right. \\
C_{p}^{*}\left(\epsilon \frac{\partial \rho^{*} U \theta}{\partial X}+\frac{\partial \rho^{*} V \theta}{\partial Y}\right)=\frac{1}{P r_{i n} R e_{i n}}\left[\epsilon^{2} \frac{\partial}{\partial X}\left(k^{*} \frac{\partial \theta}{\partial X}\right)+\frac{\partial}{\partial Y}\left(k^{*} \frac{\partial \theta}{\partial Y}\right)\right]+ \\
\frac{E c_{i n}}{R e_{i n}}\left(U \frac{\partial P}{\partial X}+\frac{1}{\epsilon} V \frac{\partial P}{\partial Y}\right)+\frac{E c_{i n}}{R e_{i n}} \Phi_{v}
\end{gathered}
$$

where $\Phi_{v}$ is the dimensionless form of the viscous dissipation term (Eq. (5)):

$$
\Phi_{v}=\mu^{*}\left\{2\left[\left(\epsilon \frac{\partial U}{\partial X}\right)^{2}+\left(\frac{\partial V}{\partial Y}\right)^{2}\right]+\left[\frac{\partial U}{\partial Y}+\epsilon \frac{\partial V}{\partial X}\right]^{2}-\frac{2}{3}\left[\epsilon \frac{\partial U}{\partial X}+\frac{\partial V}{\partial Y}\right]^{2}\right\}
$$

The equation of state (6) becomes:

$$
\rho^{*}=\frac{1+\frac{\Delta P_{r e f}}{p_{i n}} P}{1+\frac{\Delta T_{r e f}}{T_{i n}} \theta}
$$

In these equations, $R e_{i n}=\rho_{i n} \bar{u}_{i n} D_{h} / \mu_{i n}, P r_{i n}=\mu_{i n} C_{p, i n} / k_{i n}$ and $E c_{i n}=\bar{u}_{i n}^{2} / C_{p, i n} \Delta T_{r e f}$ are the Reynolds, Prandtl and Eckert numbers at the inlet section.

The energy equation (24) shows that the pressure work (PW) and viscous dissipation terms (VD) (the last two terms) are both in the order of $E c_{i n} / R e_{i n}$, considering that, from the continuity equation (21), $V \sim \mathcal{E}$ in the PW term. The ratio PW/VD is therefore of the order of -1 since PW is negative and VD is positive. Furthermore, by introducing the Mach number $M a_{i n}{ }^{2}=\rho_{i n} \bar{u}_{i n}^{2} / \gamma \mathrm{p}_{\text {in }}=\bar{u}_{i n}^{2} / \gamma r T_{\text {in }}$ and the Knudsen number $K n_{i n}=\lambda_{i n} / D_{h}$ at inlet, the sum of PW and VD is proportional to the product $M a_{i n} K n_{i n}$ since it can be shown that:

$$
\frac{E c_{i n}}{R e_{i n}}=\sqrt{\frac{2}{\pi \gamma}}(\gamma-1)\left(\frac{T_{i n}}{\Delta T_{r e f}}\right) M a_{i n} K n_{i n}
$$

Thus PW and VD can be neglected at the same time only when the gas flow is incompressible or weakly compressible $\left(M a_{i n}<<1\right)$ and for no slip gas flows $\left(K n_{i n}<<1\right)$. 
The dimensionless form of the boundary conditions is:

- at $X=0, \forall Y \in\left[0, \frac{1}{4}\right], P_{\text {in }}=0, \frac{\partial U}{\partial X}=0, V=0$ and $\theta_{\text {in }}=0$;

- at $X=1, \forall Y \in\left[0, \frac{1}{4}\right], P_{\text {out }}=\frac{p_{\text {in }}}{\Delta P_{\text {ref }}}\left(\frac{p_{\text {out }}}{p_{\text {in }}}-1\right)<0, \frac{\partial U}{\partial X}=0, V=0$ and $\frac{\partial \theta}{\partial X}=0$;

- at $Y=0, \forall X \in[0,1], \frac{\partial U}{\partial Y}=V=\frac{\partial \theta}{\partial Y}=0$;

- at $Y=\frac{1}{4}, \forall X \in[0,1]$, the velocity boundary conditions (10) and (11) write:

$$
\begin{gathered}
U_{g}=-\sigma_{\mu} K n_{i n} \frac{\mu_{g}^{*}}{\sqrt{\rho_{g}^{*}\left(1+\frac{\left.\Delta P_{\text {ref }} P_{\text {in }}\right)}{\partial Y}\right.}} \frac{\partial U}{\partial Y}+\left.\sigma_{\theta} \frac{2(\gamma-1)}{\pi \gamma} \epsilon \frac{R e_{i n} K n_{i n}^{2}}{E c_{i n}} \frac{\mu_{g}^{*}}{\left(1+\frac{\Delta P_{r e f}}{p_{\text {in }}} P_{g}\right)} \frac{\partial \theta}{\partial X}\right|_{g} \\
V_{g}=0
\end{gathered}
$$

and the thermal conditions write:

- the isothermal wall condition (13) becomes:

$$
\theta_{w}-\theta_{g}=\left.\xi_{T} K n_{i n} \frac{\mu_{g}^{*}}{\sqrt{\rho_{g}^{*}\left(1+\frac{\Delta P_{\text {ref }}}{p_{\text {in }}} P_{g}\right)}} \frac{\partial \theta}{\partial Y}\right|_{g}
$$

- the total wall heat flux $q_{w}$ defined by Eq. (14) becomes:

$$
\frac{q_{w} D_{h}}{k_{\text {in }} \Delta T_{\text {ref }}}=\left.k_{g}^{*} \frac{\partial \theta}{\partial Y}\right|_{g}+\left.P r_{i n} E c_{i n} \mu_{g}^{*} U_{g} \frac{\partial U}{\partial Y}\right|_{g}
$$

As it can be shown that:

$$
\begin{aligned}
\frac{\Delta P_{\text {ref }}}{p_{\text {in }}} & =\frac{\gamma}{\epsilon} \frac{M a_{\text {in }}^{2}}{R e_{\text {in }}} \\
\frac{\Delta T_{\text {ref }}}{T_{\text {in }}} & =(\gamma-1) \frac{M a_{\text {in }}^{2}}{E c_{\text {in }}}
\end{aligned}
$$

where the Mach number is a function of the Reynolds and Knudsen numbers :

$$
M a_{i n}^{2}=\frac{\rho_{i n} \bar{u}_{i n}^{2}}{\gamma p_{i n}}=\frac{\bar{u}_{i n}^{2}}{\gamma r T_{i n}}=\frac{2}{\pi \gamma} R e_{i n}^{2} K n_{i n}^{2}
$$

then the number of independent parameters in the above dimensionless conservation equations and boundary conditions is 10 :

$$
\operatorname{Pr}_{\text {in }}, M a_{\text {in }}, K n_{\text {in }}, \frac{\Delta T_{\text {ref }}}{T_{\text {in }}}, \frac{p_{\text {out }}}{p_{\text {in }}}, \epsilon, \sigma_{\mu}, \sigma_{\theta}, \xi_{T}, \gamma
$$

Note that due to the conservation of the mass flow rate, the Reynolds number is constant in the whole channel: $R e=R e_{i n}$. On the other hand, $M a$ and $K n$ vary along the flow such as, from Eq. (33): $M a / M a_{i n}$ $=K n / K n_{i n}$. 
Dimensional analysis of PW, VD, VSP and TC The order of magnitude of the pressure work (PW), viscous dissipation (VD), viscous stress power (VSP) and thermal creep (TC) terms in the mathematical model can be evaluated as a function of the rarefaction effect (represented by $K n_{\text {in }}$ ) and the compressibility effect (represented by $M a_{i n}$ ), for different heating rates (represented by $\Delta T_{\text {ref }} f T_{i n}$ ). This analysis is limited to subsonic low rarefied gas flows: $P r_{i n} \sim 1, M a_{i n}<1$ and $10^{-3} \leq K n_{\text {in }} \leq 0.1$, for relative pressure and temperature differences such that $\Delta P_{r e f} / p_{i n} \leq 1$ and $\Delta T_{r e f} f T_{i n} \leq 1$, where $\Delta P_{\text {ref }}$ and $\Delta T_{r e f}$ are defined by Eqs. (17) and (18). The typical micro-flows considered here are gas flows through long micro channels of hydraulic diameter $D_{h} \sim 1$ to $10 \mu \mathrm{m}$ and length $L \sim 0.1$ to $10 \mathrm{~mm}$. The inverse of the longitudinal aspect ratio is therefore a small parameter: $10^{-4} \leq \mathcal{E}=D_{h} / L \leq 0.1$.

As already discussed in Eqs. (24)-(25), PW and VD are both of the order of $E c_{i n} / R e_{i n}$. In Eq. (30), VSP is of the order of $E c_{i n} / R e_{i n}$ and, in Eq. (28), TC is of the order of $\varepsilon R e_{i n} K n_{i n}{ }^{2} / E c_{\text {in }}$ but with a quite small constant in factor of this term since $2 \sigma_{\theta}(\gamma-1) / \pi \gamma \sim 0.1$ to 0.2 . Thus, by comparing the order of magnitude of these different terms (in terms of power of the small parametere) with the other terms in the equation in which they appear, it can be shown that, whatever $K n_{i n}$ for $O\left(\varepsilon^{2}\right) \leq K n_{i n} \leq O\left(\varepsilon^{l / 2}\right)$, PW, VD and VSP are negligible for $M a_{i n} \leq O(\varepsilon)$; this approximately corresponds to $R e_{i n} \leq O(1)$. On the other hand, TC is negligible for $M a_{i n} \geq O(\varepsilon)$, that is for approximately $R e_{i n}>O(1)$.

\section{ANALYTICAL SOLUTION OF THE ASYMPTOTIC MODEL}

Solutions for the velocity and pressure fields In this section, following the asymptotic analyses by Arkilic et al. [1997] and Cai et al. [2007], analytical solutions of simplified asymptotic models for long micro channels are calculated in micro channels in the case of isothermal walls. The asymptotic analysis is carried out for $M a_{i n} \sim O(\varepsilon)$ and $\Delta T_{r e f} f T_{i n} \leq O(1)$, for $K n_{i n} \leq O\left(\varepsilon^{l / 2}\right)$. It can be shown from Eqs. (31) and (33) that this corresponds to $O\left(\varepsilon^{1 / 2}\right) \leq R e_{i n} \leq O\left(\varepsilon^{-1}\right)$ and $\Delta P_{r e f} / p_{i n} \leq O\left(\varepsilon^{1 / 2}\right)$. This parameter range is chosen because, from the previous section, the TC, PW, VD and VSP terms could potentially be negligible and simplify the solution.

Considering weakly rarefied flows with small temperature variations, the dimensionless quantities can be written as asymptotic expansion of $\varepsilon$ as follows:

$$
\begin{aligned}
U=U_{0}+\epsilon U_{1}+\epsilon^{2} U_{2}+\ldots ; & V=V_{0}+\epsilon V_{1}+\epsilon^{2} V_{2}+\ldots ; \quad P=P_{0}+\epsilon P_{1}+\epsilon^{2} P_{2}+\ldots ; \\
\theta=1+\epsilon \theta_{1}+\epsilon^{2} \theta_{2}+\ldots ; & \rho^{*}=\rho_{0}^{*}+\epsilon \rho_{1}^{*}+\epsilon^{2} \rho_{2}^{*}+\ldots
\end{aligned}
$$

For isothermal walls, the dimensionless temperature is supposed constant, equal to $\theta=1$, at the zero order of $\varepsilon$. The temperature expansions are thus compatible with flows at constant or slowly varying temperature. As a consequence and to simplify the analytical solution of the asymptotic equations, the physical properties are considered as constant:

$$
\mu_{0}^{*}=k_{0}^{*}=C_{p, 0}^{*}=1
$$

Next, the asymptotic expansions (35) are injected into the dimensionless conservation equations and boundary conditions (21)-(30). Then they can be written at the zero or first order in $\varepsilon$, and simplified by accounting for the ranges of variations of the dimensionless parameters expressed as a function of $\varepsilon$. It can be shown that the asymptotic model to compute the velocity and pressure fields of the present problem is nearly identical to the asymptotic model in the case of a fully isothermal flow solved by Arkilic et al. [1997]. Thus by noting

$$
p_{0}^{*}(X)=1+\frac{\Delta P_{\text {ref }}}{p_{\text {in }}} P_{0}(X)
$$

the state equation for the ideal gas becomes: 


$$
\rho_{0}^{*}(X)=\frac{1+\frac{\Delta P_{r e f}}{p_{i n}} P_{0}(X)}{1+\frac{\Delta T_{r e f}}{T_{i n}}}=\frac{p_{0}^{*}(X)}{1+\frac{\Delta T_{r e f}}{T_{i n}}}
$$

The analytical solutions for the velocity and pressure fields are $V_{0}=0$ and:

$$
\begin{gathered}
U_{0}(X, Y)=\frac{-1}{32} \frac{p_{\text {in }}}{\Delta P_{\text {ref }}} \frac{d p_{0}^{*}(X)}{d X}\left[1-16 Y^{2}+\frac{A}{p_{0}^{*}(X)}\right]=\frac{-1}{2} \frac{d P_{0}(X)}{d X}\left[b(X)-Y^{2}\right] \\
V_{1}(X, Y)=\frac{A}{64} \frac{p_{\text {in }}}{\Delta P_{\text {ref }}} \frac{1}{p_{0}^{*}(X)} \frac{d^{2} p_{0}^{*}(X)}{d X^{2}}\left[16 Y^{3}-Y\right]=\frac{A}{64} \frac{1}{p_{0}^{*}(X)} \frac{d^{2} P_{0}(X)}{d X^{2}}\left[16 Y^{3}-Y\right] \\
p_{0}^{*}(X)=1+\frac{\Delta P_{\text {ref }}}{p_{\text {in }}} P_{0}(X)=\left\{\left(\frac{p_{\text {out }}}{p_{\text {in }}}+\frac{3 A}{2}\right)^{2} X+\left(1+\frac{3 A}{2}\right)^{2}(1-X)\right\}^{1 / 2}-\frac{3 A}{2}
\end{gathered}
$$

with :

$$
\begin{aligned}
& b(X)=\frac{1}{16}\left[1+\frac{A}{p_{0}^{*}(X)}\right] \\
& A=8 \sigma_{\mu} K n_{\text {in }} \sqrt{1+\frac{\Delta T_{\text {ref }}}{T_{\text {in }}}}
\end{aligned}
$$

To allow the integration of the $x$-momentum equation to get the solution (39-43), the convective term and the thermal creep term were neglected. The asymptotic analysis indicates that the first approximation is a priori valid for $R e_{i n} \leq O(1)$.

Solution for the temperature field The asymptotic solution of the temperature field for an isothermal wall micro channel was computed by Cai et al. [2007]. This solution is adapted here to the present nondimensional formalism. For $R e_{i n} \leq O(1)$, it can be shown that the convective term and the PW, VD and VSP terms are all at least one order smaller than the diffusive term of the energy equation. However, the simplified problem, reduced to a pure diffusion problem is not compatible with the boundary conditions and cannot be solved. So the asymptotic thermal problem is relaxed by taking into account the PW, VD and VSP terms. Thus the energy equation for $\theta_{l}(X, Y)$ is taken as follows:

$$
\frac{\partial}{\partial Y}\left(k_{0}^{*} \frac{\partial \theta_{1}}{\partial Y}\right)=-\frac{P r_{i n} E c_{i n}}{\epsilon}\left[U_{0} \frac{\partial P_{0}}{\partial X}+\mu_{0}^{*}\left(\frac{\partial U_{0}}{\partial Y}\right)^{2}\right]
$$

The symmetry boundary condition is considered on channel axis (at $Y=0$ ) and the temperature jump condition at the wall (at $Y=1 / 4)$ :

$$
\theta_{1, g}=-\left.\xi_{T} \underbrace{K n_{\text {in }} \frac{\sqrt{1+\frac{\Delta T_{\text {ref }}}{T_{\text {in }}}}}{p_{0}^{*}(X)}}_{=K n_{g}(X)} \frac{\partial \theta_{1}}{\partial Y}\right|_{g}
$$

Then the temperature solution is given by:

$$
\begin{gathered}
\theta_{1}(X, Y)=-F(X)\left\{8 Y^{4}-16 b(X) Y^{2}+J(X)\right\} \\
F(X)=\frac{P r_{i n} E c_{i n}}{64 \epsilon}\left(\frac{d P_{0}(X)}{d X}\right)^{2} \\
b(X)=\frac{1}{16}\left[1+\frac{A}{p_{0}^{*}(X)}\right] \\
J(X)=\frac{1}{16} \frac{A}{p_{0}^{*}(X)}\left(1+\frac{B}{p_{0}^{*}(X)}\right)+\frac{1}{32}
\end{gathered}
$$




$$
\begin{aligned}
& A=8 \sigma_{\mu} K n_{\text {in }} \sqrt{1+\frac{\Delta T_{r e f}}{T_{i n}}} \\
& B=8 \xi_{T} K n_{\text {in }} \sqrt{1+\frac{\Delta T_{r e f}}{T_{i n}}}
\end{aligned}
$$

Solution for the Nusselt number For a constant wall temperature $T_{w}$, the local Nusselt number can be defined as:

$$
N u(x)=\frac{q_{w} D_{h}}{k_{i n}\left(T_{w}-\bar{T}(x)\right)}=\frac{q_{w} D_{h}}{k_{\text {in }} \Delta T_{\text {ref }}\left(\theta_{w}-\bar{\theta}(X)\right)}=\frac{-q_{w} D_{h}}{k_{i n} \Delta T_{r e f} \epsilon \overline{\theta_{1}}(X)}
$$

where, from Eq. (30), the heat flux at the walls is given by:

and

$$
\frac{D_{h}}{L_{q}}=\frac{q_{w} D_{h}}{k_{i n} \Delta T_{r e f}}=\left.\epsilon \frac{\partial \theta_{1}}{\partial Y}\right|_{g}+\left.P r_{i n} E c_{i n} U_{0, g} \frac{\partial U_{0}}{\partial Y}\right|_{g}
$$

$$
\overline{\theta_{1}}(X)=\frac{4}{\overline{U_{0}}(X)} \int_{Y=0}^{1 / 4} U_{0}(X) \theta_{1}(X, Y) d Y
$$

Therefore

$$
N u(X)=\frac{-1}{\epsilon \overline{\theta_{1}}(X)}\left(\left.\epsilon \frac{\partial \theta_{1}}{\partial Y}\right|_{g}+\left.P r_{i n} E c_{i n} U_{0, g} \frac{\partial U_{0}}{\partial Y}\right|_{g}\right)
$$

From Eq. (46), it can be shown that the conductive part of $N u(X)$ is equal to:

$$
\left.\epsilon \frac{\partial \theta_{1}}{\partial Y}\right|_{g}=\epsilon F(X) \frac{16 b(x)-1}{2}=\frac{\epsilon F(X)}{2} \frac{A}{p_{0}^{*}(X)}
$$

and, from Eq. (39), the VSP part of $N u(X)$ is equal to:

$$
\left.P r_{i n} E c_{i n} U_{0, g} \frac{\partial U_{0}}{\partial Y}\right|_{g}=\frac{-\epsilon F(X)}{2} \frac{A}{p_{0}^{*}(X)}
$$

It is remarkable that the viscous stress power at the wall is the opposite of the conduction heat flux (compare Eqs. (56) and (57)): both terms have opposite behaviours or vanish at the same time. This can be explained by the simplification of the energy equation (44) resulting from the asymptotic analysis. The convective term is not considered in (44) and, as a consequence, the thermal entrance effects and the streamwise variation of the temperature from inlet are neglected. When $K n_{\text {in }}=0$ the two terms (56) and (57) vanish because $A=0$ (see Eq. (50)). In this case, the power of the viscous stress at the wall is zero (no slip) and, as shown previously, the PW and VD terms compensate each other. Therefore, the temperature is equal to the wall temperature $T_{w}$ in the whole micro channel when $\mathrm{A}=0$. On the other hand, when $K n_{i n} \neq 0$, the conduction heat flux close to the wall and the power of the viscous stress at the wall are not zero due to the slip and the temperature jump.

The other consequence of the opposite values of (56) and (57) is that the total heat flux at the wall (Eq. (53)) and the total Nusselt number (Eq. (55)) are zero:

$$
q_{w}=0 \quad \text { and } \quad N u(X)=0
$$


That is, for rarefied forced convection in micro-channels at constant wall temperature (for $K n_{\text {in }}>0$ ), even if the conduction heat flux and the power of the viscous stress at the wall do not vanish, the total heat flux and the Nusselt number always vanish. This is valid far from the thermal entrance effects that can exist on a very short length. This asymptotic analysis could then explain the very small values of the Nusselt numbers measured by Demsis et al. [2009, 2010] in their experiments. For four rarefied gas flows (nitrogen, oxygen, argon and helium) in the ranges of Knudsen number 0.0022-0.032 and Reynolds numbers $0.13-14.7$, the measured Nusselt numbers vary between $6.2 \times 10^{-5}$ and $2.8 \times 10^{-2}$ which is four to five orders of magnitude smaller than the corresponding values in the continuum regime (when convection prevails). Till now, these very small values of the Nusselt number had never been explained theoretically because only the contribution of the diffusion heat flux at the wall was considered in the Nusselt number definition.

\section{COMPARISON OF THE ANALYTICAL AND NUMERICAL SOLUTIONS}

Numerical method. An in-house finite volume code is used to solve the full model (Eqs. (1-14)) discretized on a Cartesian grid. A second order centered scheme is used for the diffusive and convective terms because the maximum Reynolds number is $R e_{\max }<15$ and the cell Reynolds number is $R e_{\Delta x}<1$ (no numerical oscillation is observed in the discrete solutions). The discrete nonlinear steady equations are solved in a coupled way by Newton's algorithm. The mesh size on each space direction is $\mathrm{N}_{\mathrm{x}} \times \mathrm{N}_{\mathrm{y}}=6000 \times 60$ for a 100 -aspect-ratio computational domain. The mesh is uniform on $x$-direction and refined near the wall on $y$-direction, with a size ratio of two successive cells equal to 0.97 . A sensitivity analysis to mesh refinement has shown that the solutions are well converged in all simulated cases. The code has been validated through comparisons with experimental and numerical results [Tchekiken 2014, Tchekiken et al. 2013, 2014].

Studied cases To compare the analytical and numerical solutions, the numerical simulations are performed for a nitrogen flow at $T_{i n}=270 \mathrm{~K}$ in a micro-channel of length $L=100 \mu \mathrm{m}$ and height $H=3$ $\mu \mathrm{m}$, with walls at $T_{w}=300 \mathrm{~K}$. The physical properties of the gas are considered as constant. Their values considered at $T_{w}$ are gathered in Table 1 . Three cases, presented in Table 2, are simulated:

- case (a) is at low pressure and corresponds to a quasi incompressible flow but with a strong slip velocity at the walls all along the channel (relatively large $K n$ values at inlet and outlet);

- case (b) is at a large pressure difference between inlet and outlet and corresponds to a strongly compressible flow $\left(M a_{\text {out }}=0.6554\right)$ and a strong slip at channel outlet $\left(K n_{\text {out }}=0.1135\right)$;

- case (c) is at high pressure and corresponds to an incompressible flow with a very low slip along the channel (small $K n$ values at inlet and outlet).

Table 1

Nitrogen physical properties at $T_{w}=300 \mathrm{~K}$

\begin{tabular}{ccccccccc}
\hline$k$ & $C_{p}$ & $\mu \cdot 10^{5}$ & $P r$ & $\begin{array}{c}r \\
{[\mathrm{~J} / \mathrm{kg} . \mathrm{K}]}\end{array}$ & $\gamma=C_{p} / C_{v}$ & $\sigma_{\mu}$ & $\sigma_{\theta}$ & $\xi_{T}$ \\
{$[\mathrm{~W} / \mathrm{m} . \mathrm{K}]$} & {$[\mathrm{J} / \mathrm{kg} . \mathrm{K}]$} & {$[\mathrm{Pa} . \mathrm{s}]$} & & & & & \\
\hline 0.024712 & 1032.48 & 1.6588 & 0.693 & 296.8 & 1.4 & 1 & 1 & 2 \\
\hline
\end{tabular}

Table 2

Imposed inlet conditions and computed outlet conditions of each simulated case

\begin{tabular}{ccccccccc}
\hline Case & $\begin{array}{c}p_{\text {in }} \\
{[\mathrm{bar}]}\end{array}$ & $\begin{array}{c}p_{\text {out }} \\
{[\mathrm{bar}]}\end{array}$ & $\begin{array}{c}\Delta p_{\text {io }} \\
{[\mathrm{bar}]}\end{array}$ & $K n_{\text {in }}$ & $\begin{array}{c}R e_{\text {in }}= \\
\operatorname{Re}_{\text {out }}\end{array}$ & $M a_{\text {out }}$ & $K n_{\text {out }}$ & $N u_{\text {av }}$ \\
\hline (a) & 0.5 & 0.1 & 0.4 & 0.0196 & 1.038 & 0.0886 & 0.1031 & 0.108 \\
(b) & 1.5 & 0.1 & 1.4 & 0.00654 & 7.833 & 0.6554 & 0.1135 & 0.147 \\
(c) & 5 & 4.6 & 0.4 & 0.00196 & 12.05 & 0.0272 & 0.00225 & 0.221 \\
\hline
\end{tabular}


Table 3

$\mathrm{L}_{2}$-norm of the relative errors on $u, p$, and $T$ between the analytical and numerical solutions

\begin{tabular}{cccccc}
\hline Case & $\begin{array}{c}p_{\text {in }} \\
{[\text { bar }]}\end{array}$ & $\begin{array}{c}p_{\text {out }} \\
{[\text { bar }]}\end{array}$ & $\begin{array}{c}e_{u} \\
(\%)\end{array}$ & $\begin{array}{c}e_{T} \\
(\%)\end{array}$ & $\begin{array}{c}e_{p} \\
(\%)\end{array}$ \\
\hline (a) & 0.5 & 0.1 & 0.31 & 0.43 & 0.04 \\
(b) & 1.5 & 0.1 & 6.3 & 0.52 & 0.60 \\
(c) & 5 & 4.6 & 0.57 & 0.54 & 0.0007 \\
\hline
\end{tabular}

Comparisons of the velocity and temperature fields The comparison of the analytical and numerical solutions of the horizontal, $u$, and transversal, $v$, velocity components and the temperature, $T$, are presented in Figures 2-4 for the cases (a)-(c) respectively. Table 3 presents the $\mathrm{L}_{2}$-norm of the relative error on $u, p$, and $T$ between the analytical and numerical solutions. Globally, the analytical and numerical solutions for $u, p$ and $T$ are in a good accordance since the maximum relative error is $6.3 \%$ on $u$ in case (b). The absolute values and errors on $v$ are very small in the present problem. The errors are globally located at the channel entrance and exit regions: in the former case it is due to the analytical solution that does not take into account the entrance effects and, in the latter case, it is due to the zero gradient outflow boundary condition applied in the numerical simulation which is not adapted for small Reynolds numbers.

Figs 2-4 show a good agreement between the solutions except at the inlet for the temperature field and at the outlet in the compressible case (b). In the latter case, as the Reynolds and Mach number are large $\left(R e_{i n}=7.8\right.$ and $\left.M a_{\text {out }}=0.66\right)$, the validity domain of the asymptotic analysis $\left(R e_{\text {in }} \sim \mathrm{O}(1), M a_{\text {in }} \sim \mathrm{O}(\varepsilon)\right)$ is not satisfied and the analytical solution is no longer accurate. In the former case, there is a thermal entrance length in the numerical solution since the inlet temperature is $T_{i n}=270 \mathrm{~K}$, while this thermal entrance is not taken into account by the analytical solution. Using Figures 2-4 and the Nusselt number profiles, it is observed that the length of the thermal entrance is very short, equal to $3 \mathrm{H}$ at minimum in the low Reynolds number case (a) (Figure 5) and to $5 H$ at maximum in the higher Reynolds number case (c) (Figure 7).

Analysis of the Nusselt number The local wall Nusselt number, $N u=N u_{\text {diff }}+N u_{V S P}$, is defined by Eq. (55). It is the sum of two contributions: the first R.H.S term of Eq. (55), $N u_{\text {diff, }}$, is due to the diffusive flux in the wall boundary layer and the second R.H.S term, $N u_{V S P}$, is due to the power of the viscous stress at the walls. The average of $N u$ along the wall is denoted by $N u_{a v}$ and its values are given in Table 2. They are very low $\left(N u_{a v} \sim 0.1\right)$ compared with the well-known value of $N u=7.541$ calculated for a fully-established incompressible flow at large Péclet numbers between two isothermal parallel plates.

Figures 5-7 show that the absolute values of the magnitudes of both $N u_{\text {diff }}$ and $N u_{V S P}$ increase by increasing the Knudsen number (see the downstream part of Figure 5 where $K n$ increases and compare Figures 5 and 7) and increase with increasing Mach numbers (see the downstream part of Figure 6 where $M a$ increases and compare Figures 6 and 7). On the other hand, the total Nusselt number, $N u=$ $N u_{\text {diff }}+N u_{V S P}$, is very small in the whole channel except in the entrance where the flow is not locally thermally-established and close to the outlet section due to the outlet B.C. perturbations. In the channel core, $N u$ nearly vanishes because $N u_{V S P}$ is nearly the opposite of $N u_{\text {diff }}$ (note that it is "-N $N u_{d i f f "}$ " that is plotted on Figures 5-7). These graphs could then explain why a vanishing Nusselt number is measured in the experiments by Demsis et al. [2009, 2010] in case of rarefied flows at small Reynolds numbers. 


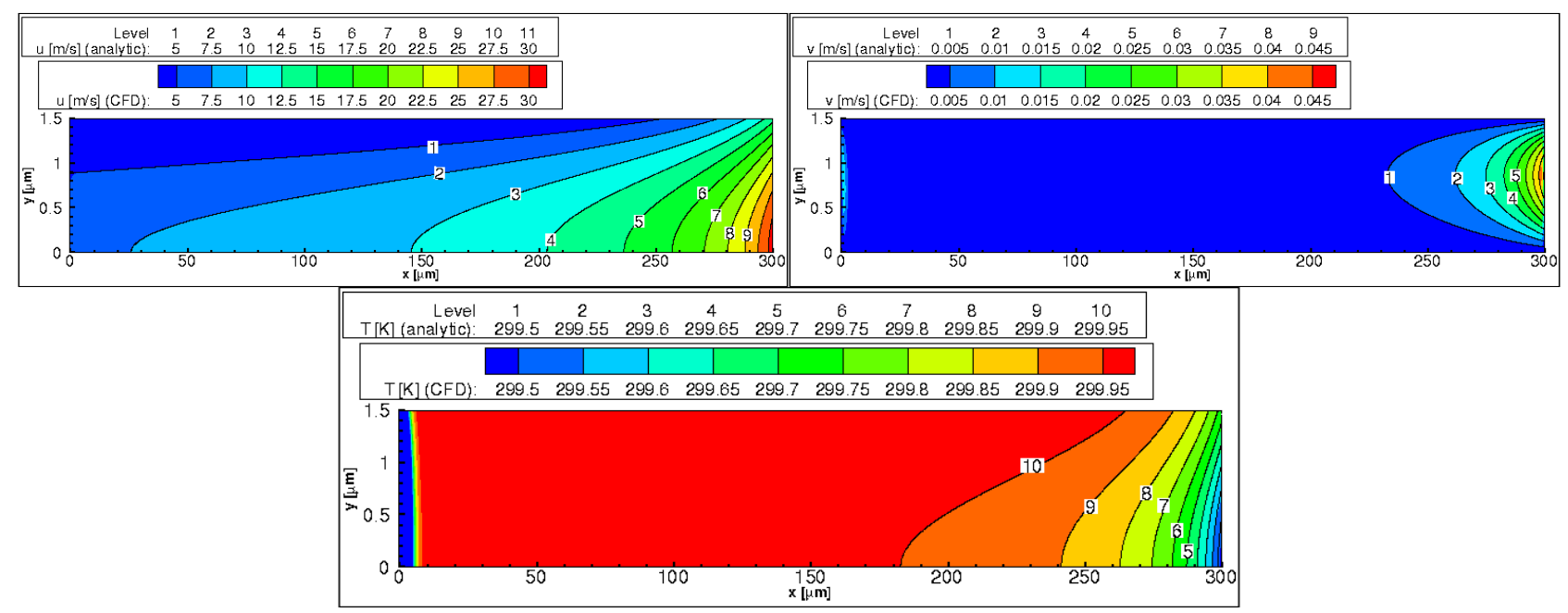

Figure 2. Comparison of the analytical solution (black isolines) and numerical solution (color fields) for $u, v$ and $T$, in case (a).

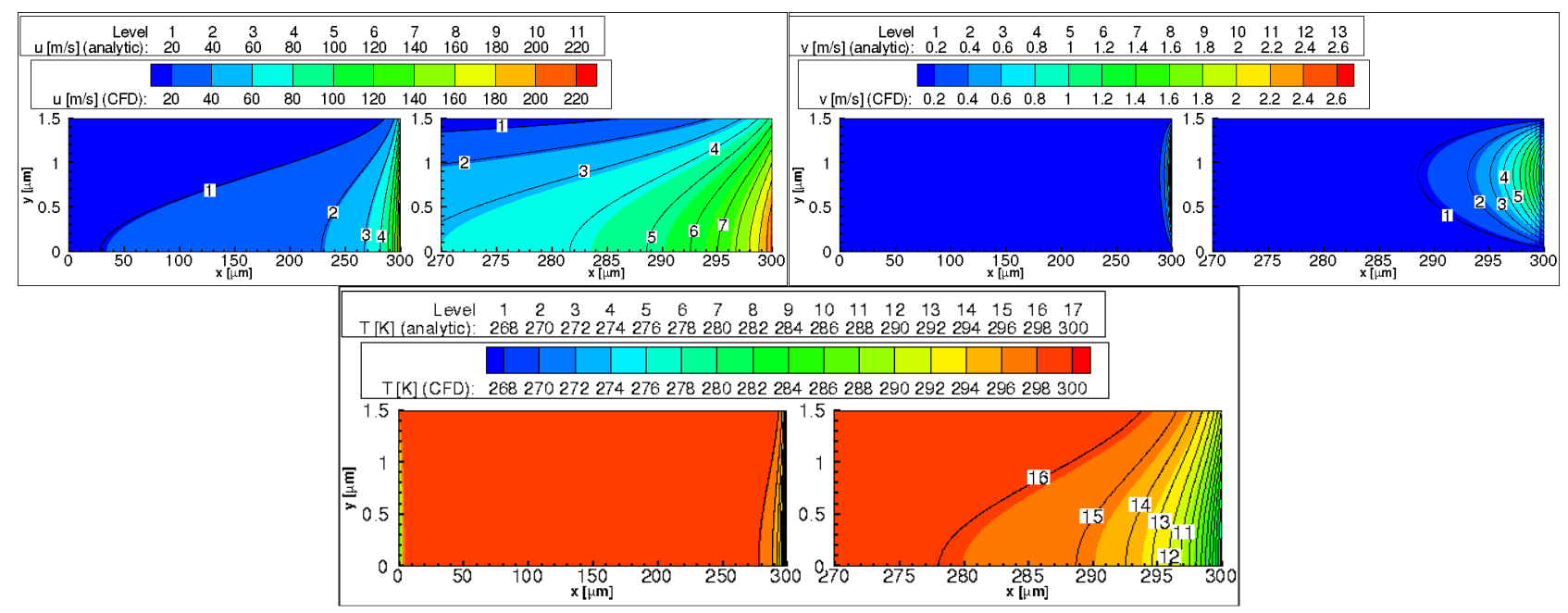

Figure 3. Same as Figure 2 but for case (b) and with a zoom on the outlet zone for each field (see coordinates on the abscissa axes).

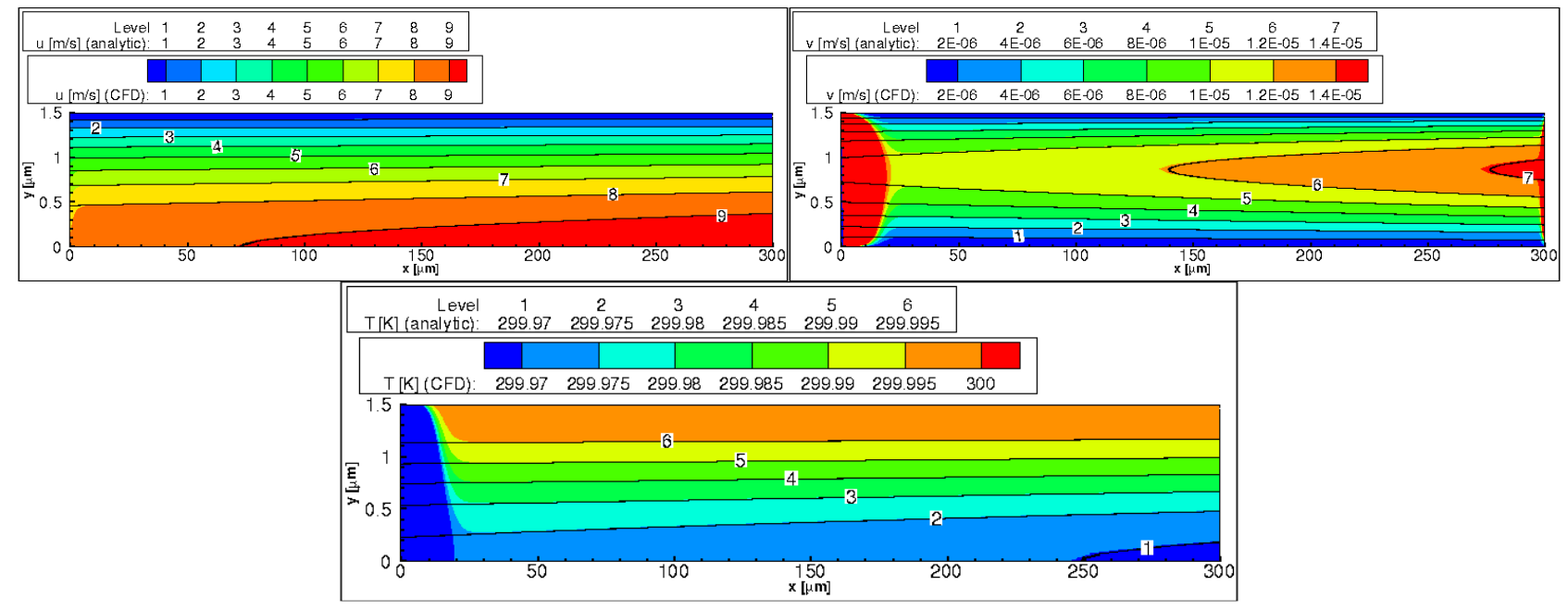

Figure 4. Same as Figure 2 but for case (c). 

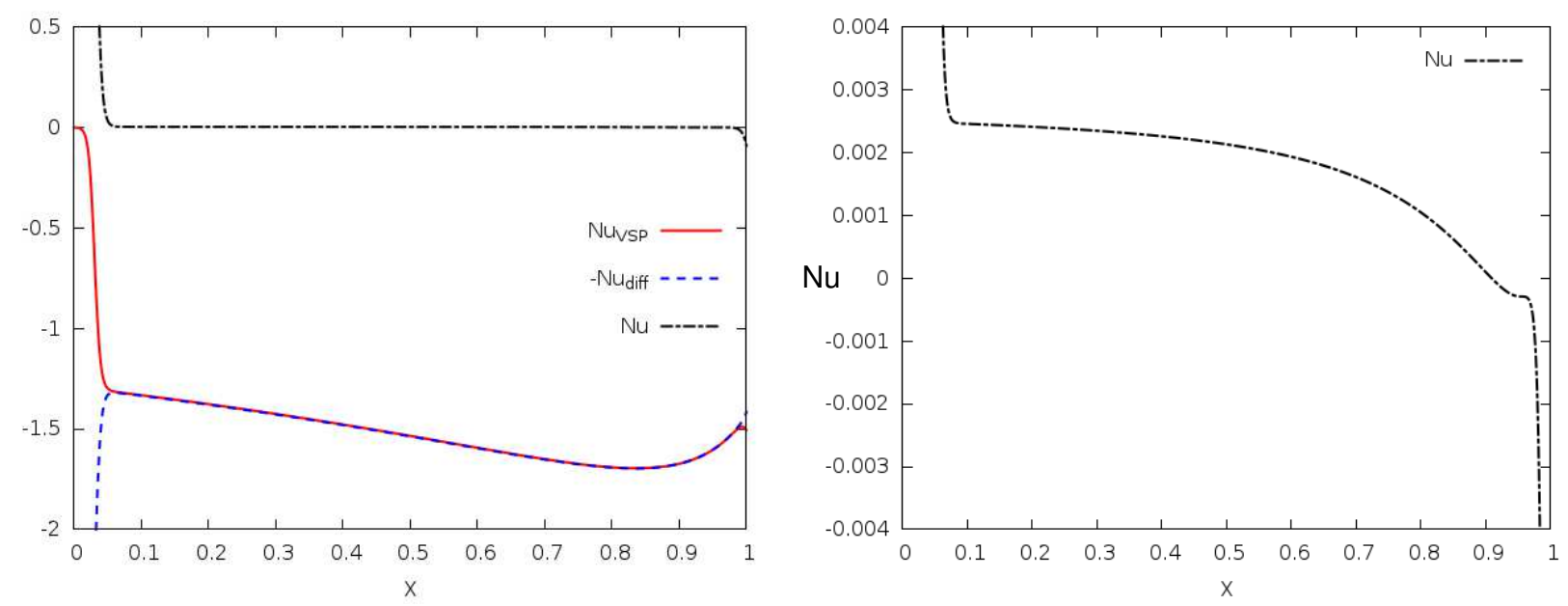

Figure 5. Profiles of the total $(N u)$, diffusive $\left(N u_{\text {diff }}\right)$ and VSP $\left(N u_{V S P}\right)$ contributions of the Nusselt number with respect to $X=x / L$, simulated in case (a) and zoom on the $N u$ profile (on the right).
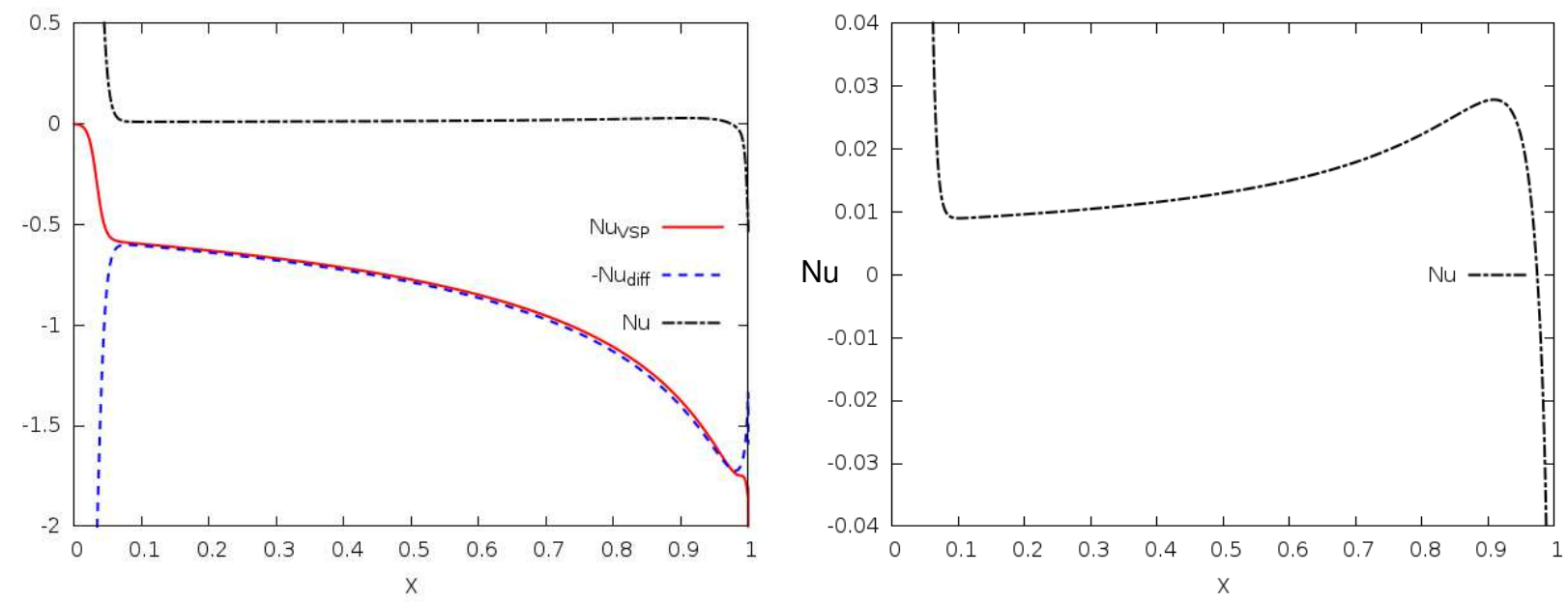

Figure 6. Profiles of the total $(N u)$, diffusive $\left(N u_{d i f f}\right)$ and VSP $\left(N u_{V S P}\right)$ contributions of the Nusselt number with respect to $X=x / L$, simulated in case (b) and zoom on the $N u$ profile (on the right).
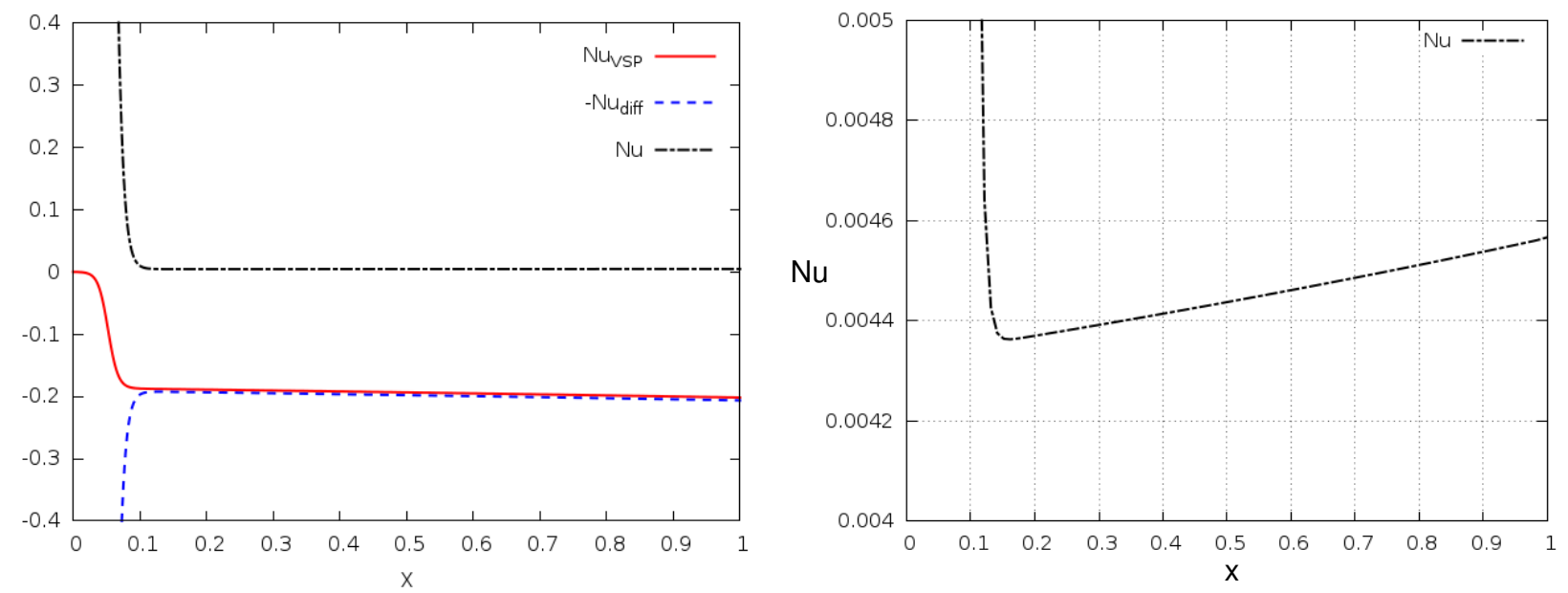

Figure 7. Profiles of the total $(N u)$, diffusive $\left(N u_{\text {diff }}\right)$ and VSP $\left(N u_{V S P}\right)$ contributions of the Nusselt number with respect to $X=x / L$, simulated in case (c) and zoom on the $N u$ profile (on the right). 


\section{CONCLUSION}

In this communication, the mathematical model usually used for weakly rarefied flows with first order slip and thermal jump at the walls has been analysed in the case of the forced convection of gas in long micro channels between two isothermal infinite plates. The order of magnitude of the pressure work (PW) and viscous dissipation (VD) in the bulk flow and the order of magnitude of the power of the viscous stress and thermal creep at the walls have been determined with respect to the flow parameters $(R e, M a, K n)$ and the small parameter $\varepsilon=D_{h} / L$. It has been shown that PW and VD have the same order of magnitude and cannot be neglected when the Mach number is high and the wall slip is important.

The analytical asymptotic solutions of the flow and thermal fields established by Cai et al. [2007] for isothermal wall micro-channels is compared with the solution of the full model by a finite volume method. This numerical solution takes into account the first order slip and thermal jump conditions at the walls, the power of the viscous forces, the thermal creep, the pressure work and the viscous dissipation. Three different flow types have been simulated: the first one is very slipping, the second highly compressible and the last one incompressible and non-slipping. In the three cases the analytical and simulated solutions agree well except the spanwise velocity due to entrance and outflow effects.

The analytical and numerical solutions are used to analyse the Nusselt number values and profiles. A thorough analysis of the total heat flux at the walls shows that the contribution of the power of the viscous stress at the walls must not be neglected because it is opposite to and of the order of magnitude of the diffusive flux at the walls. As a consequence, the total wall heat flux and Nusselt number tend to zero all the more so as rarefaction effects are important and the Reynolds number is small. This could then explain the very small values of the Nusselt number measured in the experiments by Demsis et al. (2009, 2010).

\section{REFERENCES}

Arkilic E.B., Schmidt M.A., and Breuer K.S [1997], Gaseous slip flow in long microchannels, J. Microelectromechanical Systems, Vol. 6, No. 2, pp. 167-178.

Cai C., Sun Q., and Boyd I. D. [2007], Gas flows in microchannels and microtubes, J. Fluid Mech., Vol. 589, pp. 305-314.

Demsis A., Verma B., Prabhu S. V., and Agrawal A. [2009]. Experimental determination of heat transfer coefficient in the slip regime and its anomalously low value, Phys. Review E, Vol. 80, 016311.

Demsis A., Verma B., Prabhu S. V., and Agrawal A. [2010]. Heat transfer coefficient of gas flowing in a circular tube under rarefied condition. Int. J. Thermal Sciences, Vol. 49, pp. 1994-1999.

Gad-el Hak, M. [1999], Fluid mechanics of microdevices - The Freeman scholar lecture, J. Fluids Engin., Vol. 121, No. 1, pp. 5-33.

Gupta N. K., An S., and Gianchandani Y. B. [2012], A Si-micromachined 48-stage Knudsen pump for on-chip vacuum, J. Micromech. Microengin., Vol. 22, No. 10.

Hong C., and Asako Y. [2010], Some considerations on thermal boundary condition of slip flow. Int. J. Heat Mass Transf., Vol. 53, No. 15-16, pp. 3075-3079.

Kandlikar S.G., Colin S., Peles Y., Garimella S., Pease R.F., Brandner J.J., Tuckerman D.B. [2013], Heat transfer in microchannels - 2012 status and research needs, J. Heat Transf., Vol. 135, No. 9, 091001.

Karniadakis G.E., Beskok A., and Aluru N.R. [2005], Microflows and Nanoflows - Fundamentals and Simulation, Interdisciplinary Applied Mathematics, Vol. 29, Springer New York. 
Lockerby D.A., Reese J.M., and Gallis M.A. [2005], The usefulness of higher-order constitutive relations for describing the Knudsen layer, Phys. Fluids, Vol. 17, No. 10.

Louisos W.F., and Hitt, D.L. [2012], Influence of wall heat transfer on supersonic micronozzle performance. J. of Spacecraft and Rockets, Vol. 49, No. 3, pp. 450-460.

Maslen S. H. [1958], On heat transfer in slip flow, J. Aeronautical Sciences, Vol. 25, pp. 400-401.

Sharipov F. [2011], Data on the velocity slip and temperature jump on a gas-solid interface, J. Physical and Chemical Reference Data, Vol. 40, No. 2.

Sparrow E. M., and Lin S. H. [1962], Laminar heat transfer in tubes under slip-flow conditions, J. Heat Transf., Vol. 84, No. 4, pp. 363-369.

Tchekiken C. [2014], Modélisation et simulations numériques d'écoulements compressibles dans des micro-conduites planes, PhD thesis, Univ. Paris Est Marne la Vallée, France.

Tchekiken C., Chénier E., Nicolas X., Lauriat G. [2014], A propos de la modélisation numérique de la convection de gaz en micro conduites, Congrès Français de Thermique, SFT2014, June 3-6, 2014, Lyon, France, $\mathrm{N}^{\circ} 8613,8$ pages.

Tchekiken C., Chénier E., Nicolas X., Lauriat G. [2014], On the numerical modeling of compressible forced convection of gases in micro-channels, 5th International Conference on "Heat Transfer and Fluid Flow in Microscale», HTFFM-V, April 22-25, 2014, Marseille, France, O-128-146.

Tchekiken C., Nicolas X., Baudey-Laubier L.-H., and Lauriat G. [2013], Simulation numérique d'écoulements anisothermes de gaz en micro canal : effets de compressibilité et de glissement, $21^{\text {ème }}$ Congrès Français de Mécanique, CFM2013, August 26-30, 2013, Bordeaux, France, 6 pages.

Yang Y., Morini G.L., Brandner J.J. [2014], Experimental analysis of the influence of wall axial conduction on gas-to-gas micro heat exchanger effectiveness. Int. J. Heat Mass Transf., Vol. 69, pp. 17-25.

Zhang W.-M., Meng G., and Wei X. [2012], A review on slip models for gas microflows, Microfluidics and Nanofluidics, Vol. 13, No. 6, pp. 845-882. 